\title{
Sequences of Commutator Operations
}

\author{
Erhard Aichinger • Nebojša Mudrinski
}

Received: 15 May 2012 / Accepted: 17 December 2012 / Published online: 19 January 2013

(C) The Author(s) 2013. This article is published with open access at Springerlink.com

\begin{abstract}
Given the congruence lattice $\mathbb{L}$ of a finite algebra $\mathbf{A}$ with a Mal'cev term, we look for those sequences of operations on $\mathbb{L}$ that are sequences of higher commutator operations of expansions of $\mathbf{A}$. The properties of higher commutators proved so far delimit the number of such sequences: the number is always at most countably infinite; if it is infinite, then $\mathbb{L}$ is the union of two proper subintervals with nonempty intersection.
\end{abstract}

Keywords Lattices $\cdot$ Sequences of operations $\cdot$ Commutators

Mathematics Subject Classifications (2010) Primary 06B10; Secondary 06A07 • 08A40

\section{Introduction}

It is well known that for a finite algebra with a Mal'cev term, the isomorphism type of the congruence lattice yields some information about the binary commutator operation. For example, the diamond $\mathbb{M}_{3}$ as a congruence lattice forces an algebra A with Mal'cev term to be abelian, and hence the commutator operation to satisfy $[1,1]_{\mathbf{A}}=0$. In this note, we will find conditions that force an algebra to be nilpotent. We call an algebra A a Mal'cev algebra if it has a Mal'cev term, that is, a ternary term

Supported by the Austrian Science Fund (FWF):P24077 and the Scientific Project 174018 of the Ministry of Science and Education of the Republic of Serbia.

E. Aichinger $(\bowtie) \cdot N$. Mudrinski

Institut für Algebra, Johannes Kepler Universität Linz, 4040 Linz, Austria

e-mail: erhard@algebra.uni-linz.ac.at

N. Mudrinski

Department of Mathematics and Informatics, Faculty of Sciences,

University of Novi Sad, 21000 Novi Sad, Serbia

e-mail: nmudrinski@dmi.uns.ac.rs 
operation $d$ with $d(a, b, b)=d(b, b, a)=a$ for all $a, b \in A$. Such an algebra is called nilpotent if its lower central series defined by $\gamma_{1}(\mathbf{A}):=1_{A}, \gamma_{n+1}(\mathbf{A}):=\left[1, \gamma_{n}(\mathbf{A})\right]_{\mathbf{A}}$ $(n \in \mathbb{N})$ reaches the congrence $0_{A}$ after finitely many steps, where $[.,]_{\mathbf{A}}$ denotes the commutator operation defined, e.g., in [11, Definition 4.150] or [7, Definition 3.2]; we use $\mathbb{N}$ for the set of positive natural numbers, and $\mathbb{N}_{0}$ for $\mathbb{N} \cup\{0\}$. As a consequence of the results of this note, the congruence lattice of a finite non-nilpotent Mal'cev algebra is equal to the set-theoretic union of two of its proper subintervals; hence congruence lattices that are no such union force the algebra to be nilpotent. This result is obtained by investigating the higher commutator operations as defined by [5]. Given a lattice $\mathbb{L}$, we will try to delimit the number of sequences $([.,],.[., .,],. \ldots)$ of operations on $\mathbb{L}$ that could be the sequence of higher commutator operations of some Mal'cev algebra with congruence lattice isomorphic to $\mathbb{L}$. We will see that the number of such sequences is at most countable, and we characterize when this number is finite.

This is motivated by the search for a classification of finite Mal'cev algebras modulo polynomial equivalence. We call two universal algebras polynomially equivalent if they are defined on the same universe and they have the same clone of polynomial operations. For example, for a set $M$ and its power set $P(M)$, the Boolean algebra $\left(P(M), \cap, \cup,^{\prime}\right)$ and the Boolean ring $(P(M), \Delta, \cap)$ are polynomially equivalent since the fundamental operations of each of these two algebras are polynomial operations of the other one.

There are several invariants of an algebra that depend on the clone of polynomial operations, but not on the particular choice of fundamental operations. One of these invariants is the congruence lattice, expanded with the binary commutator operation introduced in [14], cf. [7, 11]. Generalizing the binary commutator operation, A. Bulatov introduced multi-placed commutators for an algebra A [5, Definition 3]. For each $k \in \mathbb{N}$, and each $k$-tuple $\left(\alpha_{1}, \ldots, \alpha_{k}\right) \in(\operatorname{Con}(\mathbf{A}))^{k}$, he defined a congruence $\left[\alpha_{1}, \ldots, \alpha_{k}\right]_{\mathbf{A}}$ of $\mathbf{A}$ and named it the $k$-ary commutator of $\alpha_{1}, \ldots, \alpha_{k}$. When $\mathbf{A}$ has a Mal'cev term, [3,12] discuss several properties of these higher commutators. As for the binary commutator, the higher commutator operations are completely determined by the clone of polynomial functions of an algebra.

With each algebra $\mathbf{A}$, we can associate the commutator structure of $\mathbf{A}$. This is the structure $\left(\operatorname{Con}(\mathbf{A}), \wedge, \vee,\left(f_{i}\right)_{i \in \mathbb{N}}\right)$, where $f_{i}:(\operatorname{Con}(\mathbf{A}))^{i} \rightarrow \operatorname{Con}(\mathbf{A}),\left(\alpha_{1}, \ldots, \alpha_{i}\right) \mapsto$ $\left[\alpha_{1}, \ldots, \alpha_{i}\right]_{\mathbf{A}} ; f_{1}\left(\alpha_{1}\right)=\left[\alpha_{1}\right]_{\mathbf{A}}$ is defined to be $\alpha_{1}$. The sequence $\left(f_{i}\right)_{i \in \mathbb{N}}$ is then called the commutator sequence of $\mathbf{A}$. If $\mathbf{A}$ has a Mal'cev term, then $(\operatorname{Con}(\mathbf{A}), \wedge, \vee)$ is a modular lattice, and for all $n, k \in \mathbb{N}$ with $k \leq n$, and for all $\alpha_{1}, \ldots, \alpha_{n}, \beta_{1}, \ldots, \beta_{n} \in$ $\operatorname{Con}(\mathbf{A})$ and $\left\{\rho_{j} \mid j \in J\right\} \subseteq \operatorname{Con}(\mathbf{A})$, we have

- (HC1) $f_{n}\left(\alpha_{1}, \ldots, \alpha_{n}\right) \leq \bigwedge_{j=1}^{n} \alpha_{j}$.

- $(\mathrm{HC} 2)$ if $\alpha_{1} \leq \beta_{1}, \ldots, \alpha_{n} \leq \beta_{n}$, then $f_{n}\left(\alpha_{1}, \ldots, \alpha_{n}\right) \leq f_{n}\left(\beta_{1}, \ldots, \beta_{n}\right)$.

- $\quad$ (HC3) $f_{n+1}\left(\alpha_{1}, \ldots, \alpha_{n+1}\right) \leq f_{n}\left(\alpha_{2}, \ldots, \alpha_{n+1}\right)$.

- (HC4) $f_{n}\left(\alpha_{1}, \ldots, \alpha_{n}\right)=f_{n}\left(\alpha_{\sigma(1)}, \ldots, \alpha_{\sigma(n)}\right)$ for all $\sigma \in S_{n}$.

- $\quad(\mathrm{HC} 7) f_{n}\left(\alpha_{1}, \ldots, \alpha_{k-1}, \bigvee_{j \in J} \rho_{j}, \alpha_{k+1}, \ldots, \alpha_{n}\right)=$ $\bigvee_{j \in J} f_{n}\left(\alpha_{1}, \ldots, \alpha_{k-1}, \rho_{j}, \alpha_{k+1}, \ldots, \alpha_{n}\right)$.

- $\quad(\mathrm{HC} 8) f_{k}\left(\alpha_{1}, \ldots, \alpha_{k-1}, f_{n-k+1}\left(\alpha_{k}, \ldots, \alpha_{n}\right)\right) \leq f_{n}\left(\alpha_{1}, \ldots, \alpha_{n}\right)$.

Let us give a brief account on the origins of these properties: in Bulatov's paper introducing higher commutators it is claimed [5, p. 46, Proposition 1] that for an algebra in a congruence modular variety, the higher commutator operations 
satisfy ( $\mathrm{HC} 1),(\mathrm{HC} 2)$, and ( $\mathrm{HC} 4)$. Furthermore, it is claimed that the following property

- $\quad\left(\mathrm{HC}^{\prime}\right) f_{n+1}\left(\alpha_{1}, \ldots, \alpha_{n+1}\right) \leq f_{n}\left(\alpha_{1}, \ldots, \alpha_{n}\right)$

holds. No proofs of any of these properties are given. In [12, pp. 32, 33, Proposition 1.3] the second author showed that (HC1), ( $\mathrm{HC} 2)$ and (HC3) hold for every universal algebra. For the properties ( $\mathrm{HC} 4),(\mathrm{HC} 7)$ and $(\mathrm{HC} 8)$, there are results only in the case that $\mathbf{A}$ has a Mal'cev term. In the presence of a Mal'cev term (HC4), (HC7) and (HC8) are proved in [12] and [3, Proposition 6.1, Lemma 6.7, Corollary 6.15], and (HC3') is then a consequence of (HC4) and (HC3). These proofs require the existence of a Mal'cev term in an essential way, hence the validity of (HC3'), (HC4), ( $\mathrm{HC} 7),(\mathrm{HC} 8)$ outside Mal'cev algebras still remains to be explored. We note that the properties (HC5) and (HC6) listed in [3] are missing from our list here. The property (HC5) relates higher commutators to a certain centralizing relation, and (HC6) relates the commutator operations of an algebra to the commutator operations of a homomorphic image.

We call two algebras commutator equivalent if they have the same commutator structure. Since an algebra has its congruence relations and its higher commutator operations completely determined by the clone of polynomial functions, polynomially equivalent algebras are commutator equivalent. For a converse, it is open whether two finite Mal'cev algebras $\mathbf{A}$ and $\mathbf{B}$ with $\mathrm{Pol}_{3}(\mathbf{A})=\mathrm{Pol}_{3}(\mathbf{B})$ and the same commutator structure must be polynomially equivalent.

Let us now consider an arbitrary sequence $\left(f_{i}\right)_{i \in \mathbb{N}}$ of operations on a complete lattice $\mathbb{L}$ such that for each $i \in \mathbb{N}$, the function $f_{i}$ is an $i$-ary operation on $\mathbb{L}$. We will call such a sequence an operation sequence on $\mathbb{L}$, and we define the sequence $\left(f_{i}\right)_{i \in \mathbb{N}}$ to be admissible if it satisfies the properties (HC1), (HC2), (HC3), (HC4), (HC7), (HC8). In the present note we will investigate the following problem:

Given a finite modular lattice $\mathbb{L}$, how many admissible sequences can be defined on $\mathbb{L}$ ?

For a Mal'cev algebra $\mathbf{A}$, the sequence of higher commutator operations $\left(f_{i}\right)_{i \in \mathbb{N}}$ with $f_{i}\left(\alpha_{1}, \ldots, \alpha_{i}\right):=\left[\alpha_{1}, \ldots, \alpha_{i}\right]_{\mathbf{A}}$ is an admissible sequence on the lattice $\mathbb{L}:=\operatorname{Con}(\mathbf{A})$. Therefore, the number of admissible sequences on the lattice is an upper bound on the number of sequences that are commutator sequences of some Mal'cev algebra $\mathbf{B}$ with $B=A$ and $\operatorname{Con}(\mathbf{A})=\operatorname{Con}(\mathbf{B})$. A natural question is whether every admissible sequence is a commutator sequence of some algebra. More precisely, given a Mal'cev algebra $\mathbf{A}$ and an admissible sequence $\left(f_{i}\right)_{i \in \mathbb{N}}$ on $\mathbb{L}:=\operatorname{Con}(\mathbf{A})$, we ask whether there is a Mal'cev algebra $\mathbf{B}$ such that the commutator structure $\left(\operatorname{Con}(\mathbf{B}), \cap, \vee,\left(g_{i}\right)_{i \in \mathbb{N}}\right)$ with $g_{i}\left(\alpha_{1}, \ldots, \alpha_{i}\right):=\left[\alpha_{1}, \ldots, \alpha_{i}\right]_{\mathbf{B}}$ of $\mathbf{B}$ is isomorphic to $\left(\mathbb{L}, \wedge, \vee,\left(f_{i}\right)_{i \in \mathbb{N}}\right)$. We will not pursue this "representation problem" in the present note any further.

Let $\mathbb{L}$ be a complete lattice, and let $\delta, \varepsilon \in \mathbb{L}$. We say that $(\delta, \varepsilon)$ is a splitting pair of $\mathbb{L}$ if $\delta<1, \varepsilon>0$, and for all $\alpha \in \mathbb{L}$, we have $\alpha \geq \varepsilon$ or $\alpha \leq \delta$. A splitting pair is called strong if $\delta \geq \varepsilon$. The lattice $\mathbb{L}$ splits if it has a splitting pair, it splits strongly if it has a strong splitting pair. Let us remark that this splitting property has often arisen in the following context: A splitting pair $(\delta, \varepsilon)$ in the congruence lattice of an algebra $\mathbf{A}$ is a rich source of congruence preserving operations on $\mathbf{A}$ because every finitary operation that is constant on $\delta$-classes and maps into one $\varepsilon$-class is a 
congruence preserving function. $[2,8,13]$ are just three examples in the literature where the splitting property of the congruence lattice was used in this way.

Our first result provides an upper bound on the cardinality of admissible sequences.

Theorem 1.1 Let $\mathbb{L}$ be a finite modular lattice. Then the number of admissible operation sequences on $\mathbb{L}$ is finite if and only if $\mathbb{L}$ does not split strongly, and countably infinite otherwise.

The proof will be completed at the end of Section 3.

Let $\left(f_{i}\right)_{i \in \mathbb{N}}$ and $\left(g_{i}\right)_{i \in \mathbb{N}}$ be operation sequences on the lattice $\mathbb{L}$. We write $\left(f_{i}\right)_{i \in \mathbb{N}} \sqsubseteq$ $\left(g_{i}\right)_{i \in \mathbb{N}}$ if for all $i \in \mathbb{N}$ and for all $\alpha_{1}, \ldots, \alpha_{i} \in \mathbb{L}$, we have $f_{i}\left(\alpha_{1}, \ldots, \alpha_{i}\right) \leq g_{i}\left(\alpha_{1}, \ldots, \alpha_{i}\right)$. Our second result is that set of all admissible sequences on a finite lattice is at most countable and has no infinite descending chains and no infinite antichains with respect to the partial order relation $\sqsubseteq$.

Theorem 1.2 Let $\mathbb{L}$ be a finite lattice, and let $S$ be the set of all admissible operation sequences on $\mathbb{L}$. Then $S$ is at most countable, and $(S, \sqsubseteq$ ) has no infinite descending chains and no infinite antichains.

In [9], we find several properties of such orderings. Theorem 1.2 will be proved in Section 3.

\section{Preliminaries on Lattices and Ordered Sets}

By $\mathbb{B}_{2}$, we denote the two element lattice on the set $\{0,1\}$, and by $\mathbb{M}_{3}$, we denote the diamond. The lattice $\mathbb{M}_{3}$ does not split. It is easy to see that the lattices $\mathbb{B}_{2}$ and $\mathbb{M}_{2}:=\mathbb{B}_{2} \times \mathbb{B}_{2}$ split, but do not split strongly. The three element chain $\{0, \theta, 1\}$ splits strongly with splitting pair $(\theta, \theta)$.

Lemma 2.1 Let $\mathbb{L}$ be a modular lattice of finite height that does not split strongly. Then there are $n \in \mathbb{N}_{0}$ and a lattice $\mathbb{M}$ such that $\mathbb{M}$ does not split and $\mathbb{L}$ is isomorphic to $\mathbb{M} \times\left(\mathbb{B}_{2}\right)^{n}$.

Proof We proceed by induction on the height of $\mathbb{L}$. If the height is 0 , then $|\mathbb{L}|=1$ and $\mathbb{L} \cong \mathbb{L} \times\left(\mathbb{B}_{2}\right)^{0}$, so we may assume that $|\mathbb{L}|>1$. If $\mathbb{L}$ does not split, we take $\mathbb{M}:=\mathbb{L}$ and $n:=0$. Now assume that $\mathbb{L}$ has a splitting pair $(\delta, \varepsilon)$. We will first show that $\varepsilon$ is an atom of $\mathbb{L}$ : if $\varepsilon^{\prime} \in \mathbb{L}$ is such that $\varepsilon>\varepsilon^{\prime}>0$, then, since $(\delta, \varepsilon)$ is a splitting pair, we have $\varepsilon^{\prime} \leq \delta$. This implies that $\left(\delta, \varepsilon^{\prime}\right)$ is a strong splitting pair, contrary to our assumptions on $\mathbb{L}$. Thus $\varepsilon$ is an atom, and similarly, $\delta$ is a coatom of $\mathbb{L}$. Since $\mathbb{L}$ does not split strongly, we have $\delta \nsucceq \varepsilon$. Let $\mathbb{L}_{1}$ be the interval $\mathbb{I}[0, \delta]$, and let $\mathbb{L}_{2}:=\mathbb{I}[0, \varepsilon]$. By a theorem of Birkhoff [11, Theorem 2.31], the sublattice of $\mathbb{L}$ generated by $L_{1} \cup L_{2}$ is isomorphic to $\mathbb{L}_{1} \times \mathbb{L}_{2}$. But since $(\delta, \varepsilon)$ is a splitting pair, we have $(x \wedge \delta) \vee(x \wedge$ $\varepsilon)=x$ for all $x \in \mathbb{L}$. To see this, assume $x \leq \delta$. Then $(x \wedge \delta) \vee(x \wedge \varepsilon)=x \vee(x \wedge \varepsilon)=$ $x$. If $x \geq \varepsilon$, then $(x \wedge \delta) \vee(x \wedge \varepsilon)=(x \wedge \delta) \vee \varepsilon=x \wedge(\delta \vee \varepsilon)=x \wedge 1=x$. Hence the sublattice generated by $L_{1} \cup L_{2}$ is equal to $\mathbb{L}$. Thus $\mathbb{L}$ is isomorphic to $\mathbb{L}_{1} \times \mathbb{L}_{2}$. 
The lattice $\mathbb{L}_{2}$ is isomorphic to $\mathbb{B}_{2}$. The lattice $\mathbb{L}_{1}$ does not split strongly: suppose $\left(\delta_{1}, \varepsilon_{1}\right)$ is a strong splitting pair of $\mathbb{L}_{1}$. Then $\left(\left(\delta_{1}, \varepsilon\right),\left(\varepsilon_{1}, 0\right)\right)$ is a strong splitting pair of $\mathbb{L}_{1} \times \mathbb{L}_{2}$, and therefore, $\mathbb{L}$ has a strong splitting pair, a contradiction. Hence applying the induction hypothesis to $\mathbb{L}_{1}$, we obtain a lattice $\mathbb{M}$ that does not split and $n \in \mathbb{N}_{0}$ with $\mathbb{L}_{1} \cong \mathbb{M} \times \mathbb{B}_{2}^{n}$, and therefore $\mathbb{L} \cong \mathbb{L}_{1} \times \mathbb{B}_{2} \cong \mathbb{M} \times \mathbb{B}_{2}^{n+1}$.

Let $\mathbb{A}=(A, \leq)$ be a partially ordered set. We say that $\mathbb{A}$ satisfies the descending chain condition if there is no infinite descending chain $a_{1}>a_{2}>a_{3}>\ldots$. The ascending chain condition is defined dually. For $m \in \mathbb{N}$, we define a partially ordered set $\mathbb{A}^{m}=\left(A^{m}, \leq\right)$, where $\left(a_{1}, \ldots, a_{m}\right) \leq\left(b_{1}, \ldots, b_{m}\right)$ if for all $i \in\{1, \ldots, m\}$, we have $a_{i} \leq b_{i}$. For $\mathbb{A}:=\left(\mathbb{N}_{0}, \leq\right)$, the following lemma is known as Dickson's Lemma [6].

Lemma 2.2 ([9, Lemma 1.2], [1, p. 195, Example (4)]) Let $\mathbb{A}$ be a partially ordered set with the descending chain condition and no infinite antichains. Then $\mathbb{A}^{m}$ satisfies the descending chain condition and has no infinite antichains.

A subset $I$ of $\mathbb{N}_{0}^{m}$ is an upward closed set if for all $\mathbf{a} \in I$ and $\mathbf{b} \in \mathbb{N}_{0}^{m}$ with $\mathbf{a} \leq \mathbf{b}$, we have $\mathbf{b} \in I$. It is easy to see that every upward closed set $U \subseteq \mathbb{N}_{0}^{m}$ is uniquely determined by its minimal elements. Since the set of minimal elements of $U$ is an antichain, Lemma 2.2 implies that this set is finite. This establishes the following lemma.

Lemma 2.3 Let $m \in \mathbb{N}$. Then there are exactly countably infinitely many upward closed subsets of $\mathbb{N}_{0}^{m}$.

We will also use the following theorem from order theory:

Theorem 2.4 (cf. [1, Corollary 4.3], [10, Theorem 1.2]) Let $m \in \mathbb{N}$, and let $\mathcal{U}$ be the set of upward closed subsets of $\mathbb{N}_{0}^{m}$. Then the partially ordered set $(\mathcal{U}, \subseteq)$ has no infinite antichain and no infinite ascending chain.

\section{Sequences of Operations}

We start with proving Theorem 1.2, which we state again for easier reference.

Theorem 1.2 Let $\mathbb{L}$ be a finite lattice, and let $S$ be the set of all admissible operation sequences on $\mathbb{L}$. Then $S$ is at most countable, and $(S, \sqsubseteq)$ has no infinite descending chains and no infinite antichains.

Proof Let $m:=|\mathbb{L}|$, let $\left\{\alpha_{1}, \ldots, \alpha_{m}\right\}$ be the set of all elements of $\mathbb{L}$, and let $F:=$ $\left(f_{i}\right)_{i \in \mathbb{N}}$ be an admissible sequence. Then for $\left(a_{1}, \ldots, a_{m}\right) \in \mathbb{N}_{0}^{m} \backslash\{(0, \ldots, 0)\}$, we define $E\left(F,\left(a_{1}, \ldots, a_{m}\right)\right)$ by

$$
E\left(F,\left(a_{1}, \ldots, a_{m}\right)\right):=f_{j}(\underbrace{\alpha_{1}, \ldots, \alpha_{1}}_{a_{1} \text { times }}, \ldots, \underbrace{\alpha_{m}, \ldots, \alpha_{m}}_{a_{m} \text { times }}),
$$

where $j:=\sum_{k=1}^{m} a_{k}$. 
For $\alpha \in \mathbb{L}$, we define $\mathcal{R}_{F}(\alpha)$ as the subset of $\mathbb{N}_{0}^{m}$ given by

$$
\mathcal{R}_{F}(\alpha)=\left\{\left(a_{1}, \ldots, a_{m}\right) \in \mathbb{N}_{0}^{m} \backslash\{(0, \ldots, 0)\} \mid E\left(F,\left(a_{1}, \ldots, a_{m}\right)\right) \leq \alpha\right\} .
$$

Since $F$ is an admissible sequence, the properties (HC3) and (HC4) can be used to show that $\mathcal{R}_{F}(\alpha)$ is an upward closed subset of $\mathbb{N}_{0}^{m}$. Thus, to each $F$ we associate $m$ upward closed sets $\mathcal{R}_{F}\left(\alpha_{1}\right), \ldots, \mathcal{R}_{F}\left(\alpha_{m}\right)$. Let $F=\left(f_{i}\right)_{i \in \mathbb{N}}$ and $G=\left(g_{i}\right)_{i \in \mathbb{N}}$ be two admissible sequences on $\mathbb{L}$. We will now show that $F \sqsubseteq G$ if and only if for all $\alpha \in \mathbb{L}$, we have $\mathcal{R}_{G}(\alpha) \subseteq \mathcal{R}_{F}(\alpha)$. For the "only if"-direction, we let $\alpha \in \mathbb{L}$ and $\mathbf{a}=\left(a_{1}, \ldots, a_{m}\right) \in \mathcal{R}_{G}(\alpha)$. Then $E\left(G,\left(a_{1}, \ldots, a_{m}\right)\right) \leq \alpha$, and thus, since $F \sqsubseteq G$, $E\left(F,\left(a_{1}, \ldots, a_{m}\right)\right) \leq \alpha$, which implies $\left(a_{1}, \ldots, a_{m}\right) \in \mathcal{R}_{F}(\alpha)$. For the "if"-direction, we let $k \in \mathbb{N}$ and $\beta_{1}, \ldots, \beta_{k} \in \mathbb{L}$. Using the symmetry property (HC4) of $f_{k}$ and $g_{k}$, we obtain $\left(a_{1}, \ldots, a_{m}\right) \in \mathbb{N}_{0}^{m}$ such that $f_{k}\left(\beta_{1}, \ldots, \beta_{k}\right)=E\left(F,\left(a_{1}, \ldots, a_{m}\right)\right)$ and $g_{k}\left(\beta_{1}, \ldots, \beta_{k}\right)=E\left(G,\left(a_{1}, \ldots, a_{m}\right)\right)$. From the last equality, we obtain that a lies in $\mathcal{R}_{G}\left(g_{k}\left(\beta_{1}, \ldots, \beta_{k}\right)\right)$. Hence we have $\mathbf{a} \in \mathcal{R}_{F}\left(g_{k}\left(\beta_{1}, \ldots, \beta_{k}\right)\right)$, which means $E(F, \mathbf{a}) \leq$ $g_{k}\left(\beta_{1}, \ldots, \beta_{k}\right)$, and thus $f_{k}\left(\beta_{1}, \ldots, \beta_{k}\right) \leq g_{k}\left(\beta_{1}, \ldots, \beta_{k}\right)$. Denoting by $\mathcal{U}$ the set of upward closed subsets of $\mathbb{N}_{0}^{m}$, we have just proved that the set $(S$, $\sqsubseteq)$ of admissible sequences is dually order isomorphic to the set $\left\{\left(\mathcal{R}_{F}\left(\alpha_{1}\right), \ldots, \mathcal{R}_{F}\left(\alpha_{m}\right)\right) \mid F \in S\right\} \subseteq$ $\mathcal{U}^{m}$ with $\mathcal{U}$ partially ordered by inclusion. Now from Lemma 2.3, we obtain that $S$ is at most countable. By Theorem 2.4, $(\mathcal{U}, \subseteq)$ has no infinite antichain and no infinite ascending chain. Applying Lemma 2.2 to the dual of $(\mathcal{U}, \subseteq)$, we obtain that $(\mathcal{U}, \subseteq)^{m}$ satisfies the ascending chain condition and has no infinite antichains. Hence $(S, \sqsubseteq)$ satisfies the descending chain condition and has no infinite antichains.

In the next lemma, we will write the elements of the direct product $\mathbb{L}_{1} \times \mathbb{L}_{2}$ as column vectors in the form $\left(\begin{array}{l}\alpha \\ \beta\end{array}\right)$.

Lemma 3.1 Let $\mathbb{L}_{1}, \mathbb{L}_{2}$ be lattices, let $\mathbb{L}:=\mathbb{L}_{1} \times \mathbb{L}_{2}$, and let $\left(f_{i}\right)_{i \in \mathbb{N}}$ be an admissible operation sequence on $\mathbb{L}$. Then for all $n \in \mathbb{N}, \alpha_{1}, \ldots, \alpha_{n} \in \mathbb{L}_{1}, \beta_{1}, \ldots, \beta_{n} \in \mathbb{L}_{2}$, we have

$$
f_{n}\left(\left(\begin{array}{c}
\alpha_{1} \\
\beta_{1}
\end{array}\right), \ldots,\left(\begin{array}{c}
\alpha_{n} \\
\beta_{n}
\end{array}\right)\right)=f_{n}\left(\left(\begin{array}{c}
\alpha_{1} \\
0
\end{array}\right), \ldots,\left(\begin{array}{c}
\alpha_{n} \\
0
\end{array}\right)\right) \vee f_{n}\left(\left(\begin{array}{c}
0 \\
\beta_{1}
\end{array}\right), \ldots,\left(\begin{array}{c}
0 \\
\beta_{n}
\end{array}\right)\right)
$$

Proof We compute $f_{n}\left(\left(\begin{array}{c}\alpha_{1} \\ \beta_{1}\end{array}\right), \ldots,\left(\begin{array}{c}\alpha_{n} \\ \beta_{n}\end{array}\right)\right)=f_{n}\left(\left(\begin{array}{c}\alpha_{1} \\ 0\end{array}\right) \vee\left(\begin{array}{c}0 \\ \beta_{1}\end{array}\right), \ldots,\left(\begin{array}{c}\alpha_{n} \\ 0\end{array}\right) \vee\left(\begin{array}{c}0 \\ \beta_{n}\end{array}\right)\right)$. Using join distributivity (HC7), the last expression is equal to the join of $2^{n}$ expressions of the form $f_{n}\left(a_{1}, \ldots, a_{n}\right)$ with $a_{i} \in\left\{\left(\begin{array}{c}\alpha_{i} \\ 0\end{array}\right),\left(\begin{array}{c}0 \\ \beta_{i}\end{array}\right)\right\}$. If $a_{i}=\left(\begin{array}{c}\alpha_{i} \\ 0\end{array}\right)$ and $a_{j}=\left(\begin{array}{c}0 \\ \beta_{j}\end{array}\right)$, then by (HC1), $f_{n}\left(a_{1}, \ldots, a_{n}\right) \leq a_{i} \wedge a_{j}=0$. Hence $f_{n}\left(\left(\begin{array}{c}\alpha_{1} \\ \beta_{1}\end{array}\right), \ldots,\left(\begin{array}{c}\alpha_{n} \\ \beta_{n}\end{array}\right)\right)$ is equal to the join of the two summands in the right hand side of Eq. 3.2.

Lemma 3.2 Let $\mathbb{B}_{2}$ be the 2-element lattice. Then there are exactly three admissible operation sequences on $\mathbb{B}_{2}$; these are $\left(f_{i}\right)_{i \in \mathbb{N}},\left(g_{i}\right)_{i \in \mathbb{N}}$, and $\left(h_{i}\right)_{i \in \mathbb{N}}$ with $f_{n}\left(\alpha_{1}, \ldots, \alpha_{n}\right)=$ 0 for all $n \in \mathbb{N}, g_{1}(1)=1$ and $g_{i}=f_{i}$ for $i \geq 2$, and $h_{n}\left(\alpha_{1}, \ldots, \alpha_{n}\right)=\alpha_{1} \wedge \ldots \wedge \alpha_{n}$ for all $n \in \mathbb{N}$.

Proof Let $\left(s_{i}\right)_{i \in \mathbb{N}}$ be an admissible operation sequence on $\mathbb{B}_{2}$. By (HC1), we have $s_{n}\left(\alpha_{1}, \ldots, \alpha_{n}\right)=0$ if $0 \in\left\{\alpha_{1}, \ldots, \alpha_{n}\right\}$. Hence we only need to determine $s_{n}(1, \ldots, 1)$. In the case $s_{1}(1)=0$, the property (HC3) yields $s_{n}(1, \ldots, 1) \leq s_{n-1}(1, \ldots, 1) \leq$ $\cdots \leq s_{1}(1)=0$ for all $n \in \mathbb{N}$, and thus $\left(s_{i}\right)_{i \in \mathbb{N}}=\left(f_{i}\right)_{i \in \mathbb{N}}$. In the case that $s_{1}(1)=1$ and $s_{2}(1,1)=0$, we have $s_{n}(1, \ldots, 1)=0$ by repeated application of (HC3), and 
thus $\left(s_{i}\right)_{i \in \mathbb{N}}=\left(g_{i}\right)_{i \in \mathbb{N}}$. In the case $s_{1}(1)=s_{2}(1,1)=1,(\mathrm{HC} 8)$ yields $s_{n}(1, \ldots, 1) \geq$ $s_{n-1}\left(1, \ldots, 1, s_{2}(1,1)\right)=s_{n-1}(1, \ldots, 1)$ for all $n \geq 3$, and thus $\left(s_{i}\right)_{i \in \mathbb{N}}=\left(h_{i}\right)_{i \in \mathbb{N}}$.

Lemma 3.3 Let $\mathbb{L}$ be a finite lattice that does not split, let $n$ be the number of atoms of $\mathbb{L}$, and let $\left(f_{i}\right)_{i \in \mathbb{N}}$ be an admissible operation sequence on $\mathbb{L}$. Then for all $k \in \mathbb{N}$ with $k \geq n$, we have $f_{k}\left(\gamma_{1}, \ldots, \gamma_{k}\right)=0$ for all $\gamma_{1}, \ldots, \gamma_{k} \in \mathbb{L}$.

Proof If $|\mathbb{L}|=1$, the assertion clearly holds, so we assume $|\mathbb{L}| \geq 2$. Let $\alpha_{1}, \ldots, \alpha_{n}$ be all the atoms of $\mathbb{L}$. If $n=1$, then $\mathbb{L}$ splits. Therefore, $n \geq 2$. For each $i \in\{1, \ldots, n\}$, we define $A(i):=\left\{x \in \mathbb{L} \mid x \nsupseteq \alpha_{i}\right\}$. We first show that for all $i \in\{1, \ldots, n\}$, we have $\bigvee\{\alpha \mid \alpha \in A(i)\}=1$. Let $\delta:=\bigvee\{\alpha \mid \alpha \in A(i)\}$. Then for every $x \in L$, we have $x \geq \alpha_{i}$ or $x \leq \delta$. Hence if $\delta<1$, then $\left(\delta, \alpha_{i}\right)$ is a splitting pair, contradicting the assumptions. Now if $k \geq n$, using (HC3) and (HC7), we obtain

$$
\begin{aligned}
f_{k}(1, \ldots, 1) & \leq f_{n}(1, \ldots, 1)=f_{n}\left(\bigvee_{x_{1} \in A(1)} x_{1}, \ldots, \bigvee_{x_{n} \in A(n)} x_{n}\right) \\
& =\bigvee_{\left(x_{1}, \ldots, x_{n}\right) \in A(1) \times \cdots \times A(n)} f_{n}\left(x_{1}, \ldots, x_{n}\right) .
\end{aligned}
$$

We will now show that each $f_{n}\left(x_{1}, \ldots, x_{n}\right)$ is equal to 0 . Suppose $f_{n}\left(x_{1}, \ldots, x_{n}\right)>$ 0 . Then there is an atom $\alpha_{j} \in L$ with $f_{n}\left(x_{1}, \ldots, x_{n}\right) \geq \alpha_{j}$. Hence by (HC1) $\alpha_{j} \leq$ $f_{n}\left(x_{1}, \ldots, x_{n}\right) \leq x_{j}$. This contradicts $x_{j} \in A(j)$.

This lemma has a consequence for the congruence lattice of a nonnilpotent algebra. We recall that a direct product $\mathbf{B}=\mathbf{A}_{1} \times \cdots \times \mathbf{A}_{n}$ is skewfree if for every congruence relation $\beta$ of $\mathbf{B}$, there are congruences $\alpha_{1} \in$ $\operatorname{Con}\left(\mathbf{A}_{1}\right), \ldots, \alpha_{n} \in \operatorname{Con}\left(\mathbf{A}_{n}\right)$ such that for all $\left(a_{1}, \ldots, a_{n}\right),\left(b_{1}, \ldots, b_{n}\right) \in B$, we have $\left(\left(a_{1}, \ldots, a_{n}\right),\left(b_{1}, \ldots, b_{n}\right)\right) \in \beta$ if and only if $\left(a_{i}, b_{i}\right) \in \alpha_{i}$ for all $i \in\{1, \ldots, n\}$.

Corollary 3.4 Let $\mathbf{A}$ be a finite Mal'cev algebra. Then we have:

(1) If $\mathbf{A}$ is not nilpotent, then its congruence lattice $\operatorname{Con}(\mathbf{A})$ splits.

(2) If $\operatorname{Con}(\mathbf{A})$ does not split strongly, then there exist $n \in \mathbb{N}_{0}$ and algebras $\mathbf{B}, \mathbf{C}_{1}, \ldots, \mathbf{C}_{n}$ such that $\mathbf{A}$ is isomorphic to the direct product $\mathbf{B} \times \mathbf{C}_{1} \times \cdots \times \mathbf{C}_{n}$, $\mathbf{B}$ is nilpotent, each $\mathbf{C}_{i}$ is simple, and the direct product is skew-free.

\section{Proof}

(1) Assume that the lattice Con(A) does not split. Then by Lemma 3.3, there is an $n \in \mathbb{N}$ such that the $n$-ary higher commutator operation of $\mathbf{A}$ satisfies $[1, \ldots, 1]_{\mathbf{A}}=0$. By $(\mathrm{HC} 8)$ and $(\mathrm{HC} 2)$, we obtain that then the $n$-th term $\gamma_{n}$ of the lower central series of $\mathbf{A}$ satisfies $\gamma_{n}=0$. Hence $\mathbf{A}$ is nilpotent, contradicting the assumptions.

(2) We assume that the congruence lattice of $\mathbf{A}$ does not split strongly.

Then Lemma 2.1 yields an $n \in \mathbb{N}_{0}$ and a lattice $\mathbb{M}$ that does not split such that $\operatorname{Con}(\mathbf{A})$ is isomorphic via some isomorphism $\iota$ to $\mathbb{M} \times \mathbb{B}_{2}^{n}$. For $i \in\{0, \ldots, n\}$, let $v_{i}:=\iota^{-1}((1,1, \ldots, 1,0,1, \ldots, 1))$ with 0 at the $(i+1)$-th place. Using the fact that these congruences permute, we obtain (cf. [11, p. 161]) that $\mathbf{A}$ is 
isomorphic to $\prod_{i=0}^{n}\left(\mathbf{A} / v_{i}\right)$. Since $\operatorname{Con}\left(\mathbf{A} / v_{0}\right)$ is isomorphic to $\mathbb{M}$, the congruence lattice of $\mathbf{A} / v_{0}$ does not split, and hence, by the the first part of this corollary, $\mathbf{A} / v_{0}$ is nilpotent. For $i \geq 1, v_{i}$ is a coatom of $\operatorname{Con}(\mathbf{A})$ and $\mathbf{A} / v_{i}$ is simple. Hence $\mathbf{B}:=\mathbf{A} / v_{0}$ and $\mathbf{C}_{i}:=\mathbf{A} / v_{i}$ satisfy $\mathbf{A} \cong \mathbf{B} \times \prod_{i=1}^{n} \mathbf{C}_{i}$. For every $\theta \in \operatorname{Con}(\mathbf{A})$, we have $\theta=\iota^{-1}(\iota(\theta))=\iota^{-1}((\iota(\theta) \vee(0,1,1 \ldots, 1)) \wedge \ldots \wedge(\iota(\theta) \vee$ $(1,1,1, \ldots, 0)))=\left(\theta \vee v_{0}\right) \wedge \ldots \wedge\left(\theta \vee v_{n}\right)$, and therefore the direct product is skew-free by [4, Lemma IV.11.6].

Theorem 3.5 Let $\mathbb{L}$ be a finite modular lattice, and let $S$ be the set of all admissible sequences on $\mathbb{L}$. Then $S$ is infinite if and only if $\mathbb{L}$ splits strongly.

Proof Let us assume that $\mathbb{L}$ does not split strongly. Then by Lemma $2.1, \mathbb{L}$ is isomorphic to a direct product $\mathbb{M} \times \mathbb{B}_{2}^{n}$ such that $\mathbb{M}$ does not split. Now by Lemmas 3.3 and 3.2, on each of the direct factors, there are only finitely many admissible operation sequences, and thus by Lemma 3.1, $S$ is finite.

If $\mathbb{L}$ splits strongly, then we choose a strong splitting pair $(\delta, \varepsilon)$, and we define an operation sequence $\left(f_{i}\right)_{i \in \mathbb{N}}$ by $f_{1}\left(\alpha_{1}\right):=\alpha_{1}$ for all $\alpha_{1} \in \mathbb{L}$, and for $i \geq 2$, $f_{i}\left(\alpha_{1}, \ldots, \alpha_{i}\right):=0$ if there exists a $j \in\{1, \ldots, i\}$ with $\alpha_{j} \leq \delta$, and $f_{i}\left(\alpha_{1}, \ldots, \alpha_{i}\right):=\varepsilon$ else. Let $g_{i}\left(\alpha_{1}, \ldots, \alpha_{i}\right)=0$ for $i \in \mathbb{N}$. Now we show that for each $k \in \mathbb{N}$, the sequence $\left(h_{i}^{(k)}\right)_{i \in \mathbb{N}}$ defined by $h_{i}^{(k)}:=f_{i}$ for $i \leq k$ and $h_{i}^{(k)}:=g_{i}$ for $i>k$ is an admissible sequence. To this end, we first show that each $f_{i}$ satisfies (HC1). Supposing that (HC1) fails for some $\alpha_{1}, \ldots, \alpha_{i}$, we have $f_{i}\left(\alpha_{1}, \ldots, \alpha_{i}\right)=\varepsilon$ and thus $\alpha_{j} \not \leq \delta$ for all $j \in\{1, \ldots, i\}$. Thus $\alpha_{j} \geq \varepsilon$ for all $j$, and therefore $f_{i}\left(\alpha_{1}, \ldots, \alpha_{i}\right) \leq \bigwedge_{j=1}^{i} \alpha_{j}$. (HC2) and (HC4) are immediate consequences of the definitions. Now for the join distributivity (HC7) of $f_{i}$ with $i \in \mathbb{N}$, having already established (HC2) and (HC4), we only need to prove

$$
f_{i}\left(\bigvee_{j \in J} \beta_{j}, \alpha_{2}, \ldots, \alpha_{i}\right) \leq \bigvee_{j \in J} f_{i}\left(\beta_{j}, \alpha_{2}, \ldots, \alpha_{i}\right)
$$

for all families $\left\langle\beta_{j} \mid j \in J\right\rangle$ from $\mathbb{L}$. Suppose that the right hand side is 0 . Then either one of the $\alpha_{k}$ satisfies $\alpha_{k} \leq \delta$, implying that the left hand side is 0 , or all $\alpha_{k}$ satisfy $\alpha_{k} \not \delta \delta$. Then we have $\beta_{j} \leq \delta$ for all $j \in J$. This implies $\bigvee_{j \in J} \beta_{j} \leq \delta$, and therefore the left hand side is 0 as well. Hence $\left(h_{i}^{(k)}\right)_{i \in \mathbb{N}}$ satisfies (HC1), (HC2), (HC4), and (HC7). (HC3) is an immediate consequence of the definition of $\left(f_{i}\right)_{i \in \mathbb{N}}$. In order to prove (HC8), we observe that for all $i, j \in \mathbb{N}$ with $j \leq i-2$, we have $f_{j+1}\left(\alpha_{1}, \ldots, \alpha_{j}, f_{i-j}\left(\alpha_{j+1}, \ldots, \alpha_{i}\right)\right) \leq f_{j+1}\left(\alpha_{1}, \ldots, \alpha_{j}, \varepsilon\right)=0$.

Now Theorem 1.1 follows immediately from Theorems 1.2 and 3.5. As a consequence, we give an upper bound on the number of pairwise commutator inequivalent Mal'cev algebras on a finite universe.

Corollary 3.6 Let $A$ be a finite set, let I be an infinite set, let $\mathbb{L}$ be a sublattice of the lattice of equivalence relations on $A$, and let $\left(\mathbf{B}_{i}\right)_{i \in I}$ be a family of Mal'cev algebras with universe $A$ such that for each $i \in I, \operatorname{Con}\left(\mathbf{B}_{i}\right)=\mathbb{L}$, and for all $i, j \in I$ with $i \neq j$, $\mathbf{B}_{i}$ and $\mathbf{B}_{j}$ are not commutator equivalent. Then $|I| \leq \aleph_{0}$, and $\mathbb{L}$ is the union of two intervals $I[0, \delta] \cup I[\varepsilon, 1]$ with $0<\varepsilon \leq \delta<1$. 
Proof For each $i \in I$ and $j \in \mathbb{N}$, we define $h_{j}^{(i)}\left(\alpha_{1}, \ldots, \alpha_{j}\right):=\left[\alpha_{1}, \ldots, \alpha_{j}\right]_{\mathbf{B}_{i}}$. Since each $\mathbf{B}_{i}$ has a Mal'cev term, each $\left(h_{j}^{(i)}\right)_{j \in \mathbb{N}}$ is an admissible sequence. Since all $\mathbf{B}_{i}$ are commutator inequivalent, we get an infinite set of admissible sequences. Thus by Theorem 1.1, I is countably infinite and $\mathbb{L}$ splits strongly, which means exactly that there exist $\varepsilon$ and $\delta$ as in the statement of the corollary.

Acknowledgements The authors thank the referee for valuable suggestions and J. Farley for helpful discussions.

Open Access This article is distributed under the terms of the Creative Commons Attribution License which permits any use, distribution, and reproduction in any medium, provided the original author(s) and the source are credited.

\section{References}

1. Aschenbrenner, M., Hemmecke, R.: Finiteness theorems in stochastic integer programming. Found. Comput. Math. 7(2), 183-227 (2007)

2. Aichinger, E.: 2-affine complete algebras need not be affine complete. Algebra Univers. 47(4), 425-434 (2002)

3. Aichinger, E., Mudrinski, N.: Some applications of higher commutators in Mal'cev algebras. Algebra Univers. 63(4), 367-403 (2010)

4. Burris, S., Sankappanavar, H.P.: A Course in Universal Algebra. Springer New York Heidelberg Berlin (1981)

5. Bulatov, A.: On the number of finite Mal'tsev algebras. In: Contributions to general algebra, vol. 13, pp. 41-54. (Velké Karlovice, 1999/Dresden, 2000) Heyn, Klagenfurt (2001)

6. Dickson, L.E.: Finiteness of the odd perfect and primitive abundant numbers with $\mathrm{n}$ distinct prime factors. Am. J. Math. 35(4), 413-422 (1913)

7. Freese, R., McKenzie, R.N.: Commutator theory for congruence modular varieties. In: London Math. Soc. Lecture Note Ser., vol. 125. Cambridge University Press (1987)

8. Horváth, G., Mayr, P., Pongrácz, A.: Characterizing translations on groups by cosets of their subgroups. Commun. Algebra 40, 3141-3168 (2012)

9. Laver, R.: Well-quasi-orderings and sets of finite sequences. Math. Proc. Cambridge Philos. Soc. 79(1), 1-10 (1976)

10. Maclagan, D.: Antichains of monomial ideals are finite. Proc. Am. Math. Soc. 129(6), 1609-1615 (2001)

11. McKenzie, R.N., McNulty, G.F., Taylor, W.F.: Algebras, Lattices, Varieties, vol. I. Wadsworth \& Brooks/Cole Advanced Books \& Software, Monterey, California (1987)

12. Mudrinski, N.: On polynomials in Mal'cev algebras. Ph.D. thesis, University of Novi Sad. http://people.dmi.uns.ac.rs/ nmudrinski/DissertationMudrinski.pdf (2009)

13. Quackenbush, R., Wolk, B.: Strong representation of congruence lattices. Algebra Univers. 1, 165-166 (1971)

14. Smith, J.D.H.: Mal'cev varieties. In: Lecture Notes in Math., vol. 554. Springer Verlag Berlin (1976) 